\title{
Serum Carotenoid, Retinol and Tocopherol Concentrations and Risk of Cervical Cancer among Chinese Women
}

\author{
Yuan-Yuan Zhang ${ }^{1 \&}$, Ling Lu ${ }^{1,2 \&}$, Guzalnur Abliz ${ }^{1 *}$, Fatima Mijit ${ }^{1}$
}

\begin{abstract}
Background: Despite many epidemiological studies on the effects of dietary antioxidant micronutrients on risk of cervical cancer, the findings remain uncertain and little evidence is available for serum nutrient markers. The present study aimed to examine the relationship between serum carotenoid, retinol and tocopherol concentrations and risk of cervical cancer among Chinese women. Materials and Methods: We conducted a hospital-based casecontrol study in which 358 adults (158 incident cases and 200 controls) were recruited from Xinjiang, China. Serum levels of carotenoids ( $\alpha$-carotene, $\beta$-carotene, $\beta$-cryptoxanthin, lycopene and lutein/zeaxanthin), retinol, and tocopherols ( $\alpha$-tocopherol and $\gamma$-tocopherol) were assessed by reverse-phase high-performance liquid chromatography. Results: We found inverse associations between serum carotenoid ( $\alpha$-carotene, $\beta$-carotene, and lutein/zeaxanthin) and tocopherol ( $\alpha$-tocopherol) concentrations and the risk of cervical cancer after adjusting for potential confounders, but a null association for retinol. The ORs for 1-SD increase were 0.71 (95 \% CI: 0.56 $0.92 ; p=0.003)$ for total carotenoids and $0.75(95 \%$ CI: 0.60-0.94; $p=0.008)$ for total tocopherols. Conclusions: These results show that higher serum concentrations of some carotenoids and tocopherols are associated with a lower risk of cervical cancer among Chinese women.
\end{abstract}

Keywords: Carotenoids - retinol - tocopherols - serum - cervical cancer - Chinese women

Asian Pac J Cancer Prev, 16 (7), 2981-2986

\section{Introduction}

Cervical cancer is the third most common cancer in women worldwide and is the fourth common cancer in Chinese women. An estimated 65,105 new cases will developed and 31,998 deaths will occur in Chinese women in 2015 (Ferlay et al., 2008). The prevalence of cervical cancer exhibits considerable geographic and racial variation, which indicates that both genetic and environmentally factors [e.g., human papillomavirus (HPV) infection, lifestyles and dietary habits] play an important role (Ferlay et al., 2008). The only established risk factor for cervical cancer is human papillomavirus (HPV) infection (Mandelblatt et al., 2002), other potential risk factors such as diet are still poorly understood.

Regarding dietary factors, epidemiological studies has suggested that fruit and vegetable intakes were negatively associated with cervical cancer (Herrero et al., 1991; Ghosh et al., 2008; Gonzalez et al., 2011), an effect that may be owing to the various antioxidant micronutrients (e.g., carotenoids, retinol and tocopherols) rich in these foods (Donaldson, 2004). Several casecontrol studies have shown strong inverse associations between consumption of some of these antioxidant micronutrients and risk of cervical cancer (Verreault et al., 1989; Herrero et al., 1991; Ghosh et al., 2008; Tomita et al., 2010). However, no associations were found regarding beta-carotene, vitamin $\mathrm{E}$ and retinol from a prospective dietary study (Gonzalez et al., 2011). Such studies are challenging, given the difficulty of accurately estimating dietary intakes because of measurement errors and may not reflect their actual bioavailability, whereas these nutrients can be better estimated by measures of circulating blood levels. Till now, only a few studies have worked on the relationship between serum or plasma concentrations of antioxidant micronutrients and the risk of cervical cancer (Batieha et al., 1993; Lehtinen et al., 1999; Tomita et al., 2010). Two studies have provided evidence of an inverse association of serum $\alpha$-carotene, $\beta$-carotene, lycopene, or tocopherols with cervical cancer risk (Batieha et al., 1993; Tomita et al., 2010), whereas these findings were not supported by another nested case-control study (Lehtinen et al., 1999). The contradictory results probably due to small case numbers, different antioxidant micronutrient intakes, and inadequately provided information regarding potential confounding factors.

To shed more light on the role of blood biomarkers of antioxidant micronutrients in risk of cervical cancer, we analyzed serum carotenoid, retinol and tocopherol concentrations from a case-control study designed to study their effects to cervical cancer. To our knowledge, this is the first case-control study to examine serum antioxidant micronutrients and risk of cervical cancer in a Chinese population.

${ }^{1}$ Affiliated Tumor Hospital, Xinjiang Medical University, ${ }^{2}$ Maternal and Child Health Hospital, Xinjiang Uygur Autonomous Region, Urumqi,Xinjiang, People’s Republic of China*For correspondence: GuzalnurAbliz@126.com 


\section{Materials and Methods}

\section{Study population}

Patients over 18 years with newly diagnosed (within 2 months) cervical cancer were consecutively recruited to this study from March 2011 through October 2014 from the Affiliated Tumor Hospital of Xinjiang Medical University in Xinjiang, China. Case patients with histologically confirmed cervical cancer were recruited prior to initiation of radiotherapy or chemotherapy. Women with a family history of cervical cancer or cervical intraepithelial neoplasia, other major chronic diseases (e.g., diabetes, stroke and other cancers), or documented cognitive impairment that would inhibit their ability to accurately respond to a questionnaire, were excluded. Among 176 eligible cases, a total of 158 cases (squamous cell carcinoma: 134 patients; adeno/adenosquamous carcinoma: 24 patients) completed face-to-face interviews and donated blood samples.

Cervical cancer-free controls were selected from women who attended a health checkup in Maternal and Child Health Hospital of Xinjiang UYgur Autonomous Region during the same period through a variety of strategies such as flyers and referrals by doctors or nurses. The selection criteria for the controls were the same as for the cases. In addition, eligible controls should have no history of cervical cancer. Of the eligible controls, 200 completed the interviews and donated blood samples in the same time frame.

Written informed consent was obtained from each study participant before the interview. The Ethical Committee of the Affiliated Tumor Hospital of Xinjiang Medical University approved the study.

\section{Epidemiologic data}

All study participants completed a face-to-face interview using a risk factor questionnaire to obtain information on demographics, socioeconomics (ie, marital status, education level and annual family income), history of cancers in a first-degree family, life habits (ie, alcohol consumption, and smoking status), medical history (ie, multivitamin and hormone therapy use), reproductive history factors, and physical activity. Current smokers were defined as individuals who had smoked at least 1 cigarette per day during the past year; Passive smokers were defined as defined as people living in a room with someone who smokes for at least $5 \mathrm{~min} / \mathrm{d}$ every day during the past year. Information on HPV infection was also collected through medical record at the same time. Weight and height were measured after interview and body mass index (BMI) was calculated as weight in kilograms divided by the square of height in meters). Venous blood samples were drawn in the morning after an overnight fast. Serum was separated within $2 \mathrm{~h}$ of being taken and was stored at $-80^{\circ} \mathrm{C}$.

\section{Assessment of serum antioxidant micronutrient concentrations}

Serum levels of antioxidant micronutrients ( $\alpha$-carotene, $\beta$-carotene, $\beta$-cryptoxanthin, lycopene, lutein/zeaxanthin, retinol, $\alpha$-tocopherol, and $\gamma$-tocopherol) were measured by reverse-phase high-performance liquid chromatography (HPLC) as method described previously (Burri et al., 2003). In this method, the sample was analyzed with a Prodigy $5 \mu \mathrm{m}$ C18 ODS $250 \times 4.6 \mathrm{~mm}$ reversed-phase column (Phenomenex, Torrance, CA, USA). 2,6-Di-tert.butyl- $p$-cresol (Sigma, USA) was used as the internal standard. Peaks were detected at a wavelength of 452 $\mathrm{nm}$ for the carotenoids, $325 \mathrm{~nm}$ for retinol and $292 \mathrm{~nm}$ for tocopherols by a Waters 2998 diode-array detector (Waters, USA). The intra-assay coefficients of variation for these micronutrients ranged from 3.6\% ( $\alpha$-tocopherol) to $9.9 \%$ ( $\alpha$-carotene); and the interassay coefficients of variation ranged from $5.6 \%$ ( $\alpha$-tocopherol) to $12.3 \%$ ( $\alpha$-carotene).

\section{Statistical analysis}

All of the analyses were performed using SPSS version 17.0 (SPSS Inc, USA). The Pearson $\chi^{2}$ test was used to test the differences between the cases and controls for categorical data. The $\mathrm{t}$ test was used to test differences for normal distributed continuous data and the Wilcoxon rank-sum test for non-normally distributed continuous data. Odds ratios (ORs) and $95 \%$ confidence intervals (CIs) were calculated as an estimate of the relative risk. The 2-sided values of $p<0.05$ were considered statistically significant.

Unconditional logistic regression analyses were used to analyze the associations of each 1 SD increase in serum antioxidant micronutrient concentrations on the risk of cervical cancer. We used two models to calculate the risk. In model 1, we only adjusted for age, and in model 2, we further adjusted for body mass index (BMI), HPV infection status, marital status, education level, annual family income, history of cancers in a first-degree family, passive smoking, alcohol drinking, multivitamin use, hormone therapy use, menopause, age at menarche, age at first birth, and physical activity. Passive smoking was used as a covariate because few participants were smokers in our study. All of the covariates in model 2 were introduced using the forward stepwise method, and criteria for entry and non-entry of these confounders were $p<0.05$ and $p>0.10$, respectively. The final logistic regression model includes variables that were statistically significant.

We performed stratified analyses in participants with and without passive smoking $(n=147$ and $n=211$, respectively). Additionally, we performed tests for effect modification by passive smoking status and by including a multiplicative interaction term.

\section{Results}

Baseline characteristics of 158 patients with cervical cancer and 200 controls were available for this analysis (Table 1). The mean age at recruitment was 45.0 years for cases and 44.9 years for controls. There were no statistically significant differences between the cases and the controls in terms of age, BMI, marital status, annual family income, age at menarche, proportions of current smokers and drinkers, menopausal women, and hormone therapy users. Compared with controls, cases reported more likely to have higher proportions of participants with 
Serum Carotenoid, Retinol and Tocopherol Concentrations and the Risk of Cervical Cancer among Chinese Women

HPV infection, history of cancers in a first-degree family, passive smokers, and older age at first birth, but selfreported significantly lower education level, multivitamin exposures, and physical activity.

The mean values and SDs of serum antioxidant micronutrients concentrations were showed in Table 2. Cases had significant lower concentration of serum total carotenoids ( $\alpha$-carotene, $\beta$-carotene, and lutein/ zeaxanthin) and total tocopherols ( $\alpha$-tocopherol) than controls, but no difference was found for retinol ( $p=0.710)$.

Table 3 shows the associations between serum antioxidant micronutrients and the risk of cervical cancer. In age-adjusted unconditional logistic regression models, significant inverse associations were observed for serum concentrations of total carotenoids ( $\alpha$-carotene, $\beta$-carotene, lutein/zeaxanthin), and total tocopherols ( $\alpha$-tocopherol) (all $p<0.05)$, but not observed for retinol $(p=0.427)$. These associations were slightly attenuated but remained after adjustment for potential risk factors such as passive smoking status and HPV infection status. For each 1-SD increase in serum antioxidant micronutrients concentrations, the OR for cervical cancer was 0.50 (95\%CI: $0.38-0.67 ; p<0.001)$ for $\alpha$-carotene, 0.70 (95\%CI: $0.56-0.88 ; p=0.002$ ) for $\beta$-carotene, 0.75 (95\%CI: $0.59-0.95 ; p=0.015)$ for lutein/zeaxanthin, 0.71 (95\%CI: $0.56-0.92 ; p=0.003$ ) for total carotenoids, 0.73 (95\%CI: $0.58-0.91 ; p=0.006)$ for $\alpha$-tocopherol, and 0.75 (95\%CI: 0.60-0.94; $p=0.008$ ) for total tocopherols, respectively.

Stratification by passive smoking status significantly altered the direction/magnitude of the observed associations for serum antioxidant micronutrients on the risk of cervical cancer (Table 4). Generally, serum carotenoids and tocopherols appeared to be more protective in passive smokers than in non-passive smokers. The $P$ value for interactions were statistically

Table 1. Baseline Characteristics of Cervical Cancer Cases and Controls

\begin{tabular}{|c|c|c|c|}
\hline Variables & Cases & Controls & $p$ \\
\hline $\mathrm{n}$ & 158 & 200 & \\
\hline Age, years & $45.0 \pm 6.73$ & $44.9 \pm 6.66$ & 0.889 \\
\hline Body mass index, $\mathrm{kg} / \mathrm{m}^{2}$ & $22.5 \pm 4.54$ & $22.3 \pm 4.90$ & 0.670 \\
\hline HPV infection status & & & $<0.001$ \\
\hline Yes & $31(19.6)$ & $180(90.0)$ & \\
\hline No & $127(80.4)$ & $20(10.0)$ & \\
\hline Marital status, n(\%) & & & 0.194 \\
\hline Married & $124(78.5)$ & $145(72.5)$ & \\
\hline Others $^{\mathrm{a}}$ & $34(21.5)$ & $55(27.5)$ & \\
\hline Education level, n(\%) & 0.007 & & \\
\hline Elementary school or below & $89(56.3)$ & $79(39.5)$ & \\
\hline Middle school & $45(28.5)$ & $78(39.0)$ & \\
\hline High school or above & $24(15.2)$ & $43(25.1)$ & \\
\hline Annual family income (yuan), $\mathrm{n}(\%)$ & & 0.247 & \\
\hline$<10,000$ & $56(35.4)$ & $59(29.5)$ & \\
\hline $10,000-50,000$ & $69(43.7)$ & $85(42.5)$ & \\
\hline$>50,000$ & $33(20.9)$ & $56(28.0)$ & \\
\hline History of cancers in a first-degree family & & 0.033 & \\
\hline Yes & $28(17.7)$ & $20(10.0)$ & \\
\hline No & $130(82.3)$ & $180(90.0)$ & \\
\hline Smoker, $\mathrm{n}(\%)$ & & & 0.770 \\
\hline Yes & $9(5.7)$ & $10(5.0)$ & \\
\hline No & $149(94.3)$ & $190(95.0)$ & \\
\hline Passive smoker, $\mathrm{n}(\%)$ & & & 0.005 \\
\hline Yes & $78(49.4)$ & $69(34.5)$ & \\
\hline No & $80(50.6)$ & $131(65.5)$ & \\
\hline Drinker, $\mathrm{n}(\%)$ & & & 0.301 \\
\hline Yes & $15(9.5)$ & $26(13.0)$ & \\
\hline No & $143(90.5)$ & $174(87.0)$ & \\
\hline Multivitamin use, $\mathrm{n}(\%)$ & & & 0.042 \\
\hline Yes & $12(7.6)$ & $29(14.5)$ & \\
\hline No & $146(92.4)$ & $171(85.5)$ & \\
\hline Hormone therapy use, $\mathrm{n}(\%)$ & & & 0.183 \\
\hline Yes & $9(5.7)$ & $19(9.5)$ & \\
\hline No & $149(94.3)$ & $181(90.5)$ & \\
\hline Menopause & & & 0.772 \\
\hline Yes & $64(40.5)$ & 78 (39.0) & \\
\hline No & $94(59.5)$ & $122(61.0)$ & \\
\hline Age at menarche, years & $11.8 \pm 2.6$ & $12.1 \pm 2.9$ & 0.304 \\
\hline Age at first birth, years & $25.8 \pm 4.8$ & $24.7 \pm 4.1$ & 0.022 \\
\hline Physical activity, MET• h/d & $76.3(25.4,124.6)$ & $95.3(35.1,132.2)$ & 0.001 \\
\hline
\end{tabular}

*Continuous variables were described by means \pm standard deviation for normal distributed continuous data and median (25th, 75 th) for non-normally distributed continuous data, ${ }^{\mathrm{a}}$ Others includes single, divorced, or widowed women 
Table 2. Serum Carotenoid, Retinol and Tocopherol Concentrations of Cervical Cancer Cases and Controls

\begin{tabular}{lcc}
\hline Variables & Cases $(\mathrm{n}=158)$ & Controls $(\mathrm{n}=200)$ \\
\hline Carotenoids & & $p$ \\
$\alpha$-carotene, $\mu \mathrm{mol} / 1$ & $0.035 \pm 0.024$ & $0.046 \pm 0.037$ \\
$\beta$-carotene, $\mu \mathrm{mol} / \mathrm{l}$ & $0.448 \pm 0.327$ & $0.596 \pm 0.314$ \\
$\beta$-cryptoxanthin, $\mu \mathrm{mol} / 1$ & $0.082 \pm 0.062$ & $0.089 \pm 0.067$ \\
Lycopene, $\mu \mathrm{mol} / \mathrm{l}$ & $0.091 \pm 0.075$ & $0.107 \pm 0.085$ \\
Lutein/zeaxanthin, $\mu \mathrm{mol} / 1$ & $0.422 \pm 0.235$ & $0.487 \pm 0.239$ \\
Total carotenoids, $\mu \mathrm{mol} / 1$ & $1.08 \pm 0.61$ & $1.31 \pm 0.78$ \\
Retinol & & 0.307 \\
Retinol, $\mu \mathrm{mol} / \mathrm{l}$ & $2.56 \pm 0.72$ & 0.010 \\
Tocopherols & & 0.002 \\
$\alpha$-tocopherol, $\mu \mathrm{mol} / \mathrm{l}$ & $34.8 \pm 13.7$ & 0.710 \\
$\gamma$-tocopherol, $\mu \mathrm{mol} / \mathrm{l}$ & $4.01 \pm 2.96$ & $39.4 \pm 14.2$ \\
Total tocopherols, $\mu \mathrm{mol} / 1$ & $38.8 \pm 14.5$ & $4.05 \pm 3.08$ \\
\hline
\end{tabular}

Table 3. Odds ratio (95\% CIs) of cervical cancer for $1 \mathrm{SD}$ increase in serum antioxidant concentrations

\begin{tabular}{|c|c|c|c|c|}
\hline \multirow[t]{2}{*}{ Serum antioxidant nutrients, $1 \mathrm{SD}$} & \multicolumn{2}{|c|}{ Model 1} & \multicolumn{2}{|c|}{ Model 2} \\
\hline & OR $(95 \% \mathrm{CI})$ & $p$ & OR $(95 \% \mathrm{CI})$ & $p$ \\
\hline \multicolumn{5}{|l|}{ Carotenoids } \\
\hline$\alpha$-carotene, $0.037 \mu \mathrm{mol} / 1$ & $0.58(0.44,0.75)$ & $<0.001$ & $0.50(0.38,0.67)$ & $<0.001$ \\
\hline$\beta$-carotene, $0.314 \mu \mathrm{mol} / 1$ & $0.67(0.54,0.84)$ & $<0.001$ & $0.70(0.56,0.88)$ & 0.002 \\
\hline$\beta$-cryptoxanthin, $0.067 \mu \mathrm{mol} / 1$ & $0.90(0.71,1.10)$ & 0.415 & $0.93(0.72,1.13)$ & 0.491 \\
\hline Lycopene, $0.085 \mu \mathrm{mol} / 1$ & $0.81(0.67,1.01)$ & 0.056 & $0.84(0.69,1.04)$ & 0.070 \\
\hline Lutein/zeaxanthin, $0.239 \mu \mathrm{mol} / 1$ & $0.75(0.60,0.94)$ & 0.013 & $0.75(0.59,0.95)$ & 0.015 \\
\hline Total carotenoids, $0.78 \mu \mathrm{mol} / 1$ & $0.70(0.56,0.89)$ & 0.002 & $0.71(0.56,0.92)$ & 0.003 \\
\hline \multicolumn{5}{|l|}{ Retinol } \\
\hline Retinol, $0.80 \mu \mathrm{mol} / 1$ & $0.91(0.73,1.14)$ & 0.427 & $0.97(0.77,1.23)$ & 0.816 \\
\hline \multicolumn{5}{|l|}{ Tocopherols } \\
\hline$\alpha$-tocopherol, $14.2 \mu \mathrm{mol} / \mathrm{l}$ & $0.71(0.57,0.89)$ & 0.002 & $0.73(0.58,0.91)$ & 0.006 \\
\hline$\gamma$-tocopherol, $3.08 \mu \mathrm{mol} / 1$ & $0.99(0.85,1.19)$ & 0.869 & $1.00(0.86,1.21)$ & 0.942 \\
\hline Total tocopherols, $13.9 \mu \mathrm{mol} / \mathrm{l}$ & $0.73(0.59,0.90)$ & 0.003 & $0.75(0.60,0.94)$ & 0.008 \\
\hline
\end{tabular}

*SD: standard deviation; OR: odds ratios; CI: confidence interval; Model 1 and model 2: Odds ratios (95\% confidence interval) from unconditional logistic model. Model 1: covariates adjusted for age; Model 2: covariates adjusted for age, BMI, HPV infection status, marital status, education level, annual family income, history of cancers in a first-degree family, passive smoking, alcohol drinking, multivitamin use, hormone therapy use, menopause, age at menarche, age at first birth, and physical activity

Table 4. Odds Eatio (95\% CIs) of Cervical Cancer for 1 SD increase in Serum Antioxidant Nutrients Stratified by Passive Smoking Status

\begin{tabular}{|c|c|c|c|c|c|}
\hline \multirow[t]{2}{*}{ Serum antioxidant nutrients, 1SD } & \multicolumn{2}{|c|}{ Passive smoker } & \multicolumn{2}{|c|}{ Non-passive smoker } & \multirow[t]{2}{*}{$p_{\text {-interactior }}$} \\
\hline & OR $(95 \% \mathrm{CI})$ & $p$ & OR $(95 \% \mathrm{CI})$ & $p$ & \\
\hline \multicolumn{6}{|l|}{ Carotenoids } \\
\hline$\alpha$-carotene, $0.037 \mu \mathrm{mol} / 1$ & $0.42(0.26,0.66)$ & $<0.001$ & $0.73(0.52,1.03)$ & 0.074 & 0.095 \\
\hline$\beta$-carotene, $0.314 \mu \mathrm{mol} / 1$ & $0.31(0.20,0.47)$ & $<0.001$ & $0.75(0.52,1.07)$ & 0.108 & 0.002 \\
\hline$\beta$-cryptoxanthin, $0.067 \mu \mathrm{mol} / \mathrm{l}$ & $0.80(0.61,1.05)$ & 0.069 & $1.05(0.82,1.29)$ & 0.822 & 0.759 \\
\hline Lycopene, $0.085 \mu \mathrm{mol} / 1$ & $0.59(0.38,0.75)$ & 0.001 & $1.02(0.79,1.22)$ & 0.831 & 0.007 \\
\hline Lutein/zeaxanthin, $0.239 \mu \mathrm{mol} / 1$ & $0.53(0.35,0.79)$ & 0.003 & $0.86(0.64,1.16)$ & 0.205 & 0.167 \\
\hline Total carotenoids, $0.78 \mu \mathrm{mol} / \mathrm{l}$ & $0.50(0.30,0.71)$ & $<0.001$ & $0.90(0.73,1.18)$ & 0.498 & 0.010 \\
\hline \multicolumn{6}{|l|}{ Retinol } \\
\hline Retinol, $0.80 \mu \mathrm{mol} / 1$ & $0.86(0.61,1.23)$ & 0.411 & $0.92(0.68,1.24)$ & 0.581 & 0.864 \\
\hline \multicolumn{6}{|l|}{ Tocopherols } \\
\hline$\alpha$-tocopherol, $14.2 \mu \mathrm{mol} / \mathrm{l}$ & $0.36(0.24,0.54)$ & $<0.001$ & $0.86(0.61,1.20)$ & 0.391 & 0.002 \\
\hline$\gamma$-tocopherol, $3.08 \mu \mathrm{mol} / \mathrm{l}$ & $0.85(0.61,1.22)$ & 0.425 & $1.08(0.94,1.35)$ & 0.553 & 0.443 \\
\hline Total tocopherols, $13.9 \mu \mathrm{mol} / 1$ & $0.39(0.26,0.58)$ & $<0.001$ & $0.90(0.63,1.28)$ & 0.755 & 0.003 \\
\hline
\end{tabular}

*SD: standard deviation; OR: odds ratios; CI: confidence interval; Covariates adjusted: as shown in table 1 (model 2)

significant for $\beta$-carotene $\left(p_{\text {-interaction }}=0.002\right)$, lycopene $\left(p_{\text {-interaction }}=0.007\right)$, total carotenoids $\left(p_{\text {-interaction }}=0.010\right)$, $\alpha$-tocopherol $\left(p_{\text {-interaction }}=0.002\right)$, and total tocopherols $\left(p_{\text {-interaction }}=0.003\right)$.

\section{Discussion}

In this hospital-based case-control study, we evaluated whether a higher concentration of antioxidant micronutrients was associated with lower risk of cervical cancer. Our results showed inverse associations between serum carotenoids ( $\alpha$-carotene, $\beta$-carotene, and lutein/ zeaxanthin) and tocopherols ( $\alpha$-tocopherol) concentrations and the risk of cervical cancer, but null association was observed for retinol.

In agreement with our findings, two case-control 
studies have found serum levels of carotenoids or tocopherols, but not retinol, to be inversely associated with the risk of cervical cancer. A hospital-based case-control study of 108 low-income Brazilian women with invasive cervical cancer showed inverse associations for vitamin E (Tomita et al., 2010). A population-based case-control study (Cho et al., 2009) that included 160 cases and 378 controls reported that serum $\beta$-carotene and vitamin E, but not retinol, were inversely associated with cervical cancer risk after adjusting for confounding factors similar to those in the present study. However, a population-based nested case-control study in which 50 women who developed cervical cancer or carcinoma-in situ over a 15 -year period reported that significantly lower risk of cervical cancer was observed for $\alpha$-carotene and $\beta$-carotene, but not for retinol, vitamin C and E (Batieha et al., 1993). And a nested casecontrol study conducted in the Finland and Sweden (38 cases and 85 controls) observed no associations for retinol and vitamin E (Lehtinen et al., 1999). Results from our study were consistent the findings from studies with larger sample size (Cho et al., 2009; Tomita et al., 2010) and found that each $1 \mathrm{SD}$ increase in $\alpha$-carotene, $\beta$-carotene, lutein/zeaxanthin, total carotenoids, $\alpha$-tocopherol, and total tocopherols was associated with a $50 \%, 30 \%, 25 \%$, $29 \%, 27 \%$ and $25 \%$ decreased risk of cervical cancer. To our knowledge, this is the first observational study to have evaluated the effect of low concentrations of different antioxidant micronutrients on cervical cancer risk in Chinese women. We used objective measures for the evaluation of both the exposure (concentration of micronutrients). Hence, our results are not biased by self-report.

There are several potential pathways that may explain the effects of carotenoids or tocopherols in preventing against the development of cervical carcinogenesis. These micronutrients have been suggested to be cancer preventive mainly due to their antioxidant properties (Sporn and Roberts, 1983). It is well known that oxidative stress plays a key role in carcinogenesis (Klaunig and Kamendulis, 2004). Carotenoids or tocopherols are very efficient physical and chemical quenchers of singlet oxygen (1O2) and potent scavengers of free radicals and oxidants to prevent against free-radical damage to DNA (Salganik, 2001). Besides direct damage nuclear and/or mitochondrial DNA, interference with oxidative DNA repair mechanisms (in both nuclear and mitochondrial compartments) also contributes to increase mutation frequency and persistent oxidative DNA damage (Klaunig and Kamendulis, 2004). Reactive oxygen species and cellular redox status could mediate cell signaling pathways on cell proliferation and apoptosis through the activation of transcription factors including mitogen-activated protein (MAP), kinase/AP-1, and $\mathrm{NF}-x \mathrm{~B}$ pathways, and lead to carcinogenesis (Klaunig and Kamendulis, 2004). Furthermore, carotenoids and tocopherols also may protect the immune system through inhibiting C-reactive protein, leucocytes, fibrinogen, and inflammatory cytokines such as IL-6, IL-1 $\beta$ and TNF- $\alpha$, and increased cytotoxic activities in tumor models, and increased killer cell function, because immune system is particularly vulnerable to oxidative stress (Gravitt et al.,
1998; McCullough and Giovannucci, 2004; Stebbing and Hart, 2011).

In this study, generally, higher levels of carotenoids and tocopherols were associated with significantly protective effects in passive smokers, but not in nonpassvie smokers. However, after excluding smokers, results were minimally attenuated which was probably due to few number of smokers (9 cases and 10 controls) in our study. Epidemiologic evidence has established that exposure to cigarette smoke dramatically increased the incidence of cancers, including cervical cancer, but the mechanism is still poorly understood (Carbone, 1992; Appleby et al., 2006). It has been suggested that a modifying role for antioxidant micronutrients by cigarette smoking could be involved in this processes (Handelman et al., 1996). Studies have shown that smoking could significantly destruction of tocopherols and carotenoids in human plasma (Handelman et al., 1996). Therefore, even if smoking does not lower blood levels of tocopherols and carotenoids, it may increase tocopherols and carotenoids turnover and decrease stores in cervical tissues. Thus, lower levels of tocopherols and carotenoids might be harmful in this condition. Studies also showed that these antioxidant micronutrients could block nitrosamine, which is the main cause of oxidative dress for smokers (Byers and Guerrero, 1995; Camphausen et al., 2005).

Potential limitations warrant comment in this study. First, the present study is an hospital-based sample, raising potential concerns about the generalizability of our findings. However, the use of hospital controls tend to increase the comparability of information obtained by cases and controls, and thus underestimate the true associations (B et al., 1996). Second, the associations derived from a case-control study might be unable to infer causality. Because data on serum markers were detected just at the time of diagnosis of cervical cancer, case subjects may have not changed their diets and thus the serum markers could also not change significantly. Third, our analyses were based on single measurements of serum antioxidant micronutrient concentrations. However, a single assessment may be susceptible to a large random error and short-term fluctuation and attenuate associations. Fourth, although we adjusted for several lifestyle factors known to potentially affect cervical cancer risk, we could not rule out the residual confounding in observational study due to some unmeasured lifestyle factors. Finally, there were few smokers in our study. Therefore, we did not have enough power to examine the association between antioxidant micronutrients and cervical cancer risk among smokers.

In conclusion, our study provides evidence that high concentrations of carotenoids ( $\alpha$-carotene, $\beta$-carotene, and lutein/zeaxanthin) and tocopherols ( $\alpha$-tocopherol) are associated with a low risk of cervical cancer, especially for individuals exposing to passive smoking. Although the findings from this epidemiological study cannot establish causality, they provide a solid base that low concentration of carotenoids and tocopherols contributes to the development of cervical carcinogenesis. Welldesigned cohort studies or clinical trials may be warranted to confirm these beneficial associations. 


\section{Acknowledgements}

We thank the doctors and nurses in the abovementioned hospitals for providing facilities to carry out the research work. This study was supported by the National Natural Science Foundation of China (No.81272335) .

\section{References}

Appleby P, Beral V, Berrington de Gonzalez A, et al (2006). Carcinoma of the cervix and tobacco smoking: collaborative reanalysis of individual data on 13,541 women with carcinoma of the cervix and 23,017 women without carcinoma of the cervix from 23 epidemiological studies. Int J Cancer, 118, 1481-95.

D’ Avanzo B, La Vecchia C, Katsouyanni K, Negri E, Trichopoulos D. (1996). Reliability of information on cigarette smoking and beverage consumption provided by hospital controls. Epidemiology, 7, 312-5.

Batieha AM, Armenian HK, Norkus EP, et al (1993). Serum micronutrients and the subsequent risk of cervical cancer in a population-based nested case-control study. Cancer Epidemiol Biomarkers Prev, 2, 335-9.

Burri BJ, Dopler-Nelson M, Neidllinger TR (2003). Measurements of the major isoforms of vitamins A and $\mathrm{E}$ and carotenoids in the blood of people with spinal-cord injuries. J Chromatogr A, 987, 359-66.

Byers T, Guerrero N (1995). Epidemiologic evidence for vitamin $\mathrm{C}$ and vitamin $\mathrm{E}$ in cancer prevention. Am J Clin Nutr, 62, 1385-92.

Camphausen K, Citrin D, Krishna MC, et al (2005). Implications for tumor control during protection of normal tissues with antioxidants. J Clin Oncol, 23, 5455-7.

Carbone D (1992). Smoking and cancer. Am J Med, 93, 13S-7S.

Cho H, Kim MK, Lee JK, et al (2009). Relationship of serum antioxidant micronutrients and sociodemographic factors to cervical neoplasia: a case-control study. Clin Chem Lab Med, 47, 1005-12.

Donaldson MS (2004). Nutrition and cancer: a review of the evidence for an anti-cancer diet. Nutr J, 3, 19.

Ferlay J, Shin HR, Bray F, et al (2008). GLOBOCAN 2008 v2.0, Cancer Incidence and Mortality Worldwide: IARC CancerBase No. 10 [Internet]. In: Lyon, France: International Agency Res Cancer; 2010. Available from: http://globocan. iarc.fr, accessed on day/month/year.

Ghosh C, Baker JA, Moysich KB, et al (2008). Dietary intakes of selected nutrients and food groups and risk of cervical cancer. Nutr Cancer, 60, 331-41.

Gonzalez CA, Travier N, Lujan-Barroso L, et al (2011). Dietary factors and in situ and invasive cervical cancer risk in the European prospective investigation into cancer and nutrition study. Int J Cancer, 129, 449-59.

Gravitt PE, Peyton CL, Apple RJ, et al (1998). Genotyping of 27 human papillomavirus types by using L1 consensus PCR products by a single-hybridization, reverse line blot detection method. J Clin Microbiol, 36, 3020-7.

Handelman GJ, Packer L, Cross CE (1996). Destruction of tocopherols, carotenoids, and retinol in human plasma by cigarette smoke. Am J Clin Nutr, 63, 559-65.

Herrero R, Potischman N, Brinton LA, et al (1991). A casecontrol study of nutrient status and invasive cervical cancer. i. dietary indicators. Am J Epidemiol, 134, 1335-46.

Klaunig JE, Kamendulis LM (2004). The role of oxidative stress in carcinogenesis. Annu Rev Pharmacol Toxicol, 44, 239-67.

Lehtinen M, Luostarinen T, Youngman LD, et al (1999). Low levels of serum vitamins $\mathrm{A}$ and $\mathrm{E}$ in blood and subsequent risk for cervical cancer: interaction with HPV seropositivity. Nutr Cancer, 34, 229-34.

Mandelblatt JS, Lawrence WF, Womack SM, et al (2002). Benefits and costs of using HPV testing to screen for cervical cancer. JAMA, 287, 2372-81.

McCullough ML, Giovannucci EL (2004). Diet and cancer prevention. Oncogene, 23, 6349-64.

Salganik RI (2001). The benefits and hazards of antioxidants: controlling apoptosis and other protective mechanisms in cancer patients and the human population. J Am Coll Nutr, 20, 464-72.

Sporn MB, Roberts AB (1983). Role of retinoids in differentiation and carcinogenesis. Cancer Res, 43, 3034-40.

Stebbing J, Hart CA (2011). Antioxidants and cancer. Lancet Oncol, 12, 996.

Tomita LY, Longatto Filho A, Costa MC, et al (2010). Diet and serum micronutrients in relation to cervical neoplasia and cancer among low-income Brazilian women. Int J Cancer, 126, 703-14.

Verreault R, Chu J, Mandelson M, et al (1989). A case-control study of diet and invasive cervical cancer. Int J Cancer, 43, 1050-4. 